\title{
Detection of Human Pegivirus (HPgV) Infection among Filipino Children with Decompensated Liver Disease Secondary to Biliary Cirrhosis and Liver Transplant Pediatric Patients
}

\author{
Inah Marie C. Aquino, ${ }^{1}$ Leslie Michelle M. Dalmacio ${ }^{2}$ and Germana Emerita V. Gregorio ${ }^{3}$ \\ ${ }^{1}$ College of Medicine, University of the Philippines Manila \\ ${ }^{2}$ Department of Biochemistry and Molecular Biology, College of Medicine, University of the Philippines Manila \\ ${ }^{3}$ Department of Pediatrics, College of Medicine and Philippine General Hospital, University of the Philippines Manila
}

\begin{abstract}
Background. Human Pegivirus (HPgV), previously called Hepatitis G virus or GB virus C, is an RNA virus. It can be transmitted vertically (mother to infant), parenterally and sexually. HPgV share common routes of transmission to other viruses such as Hepatitis B virus, Hepatitis C virus and Human Immunodeficiency virus (HIV) thus co-infection is usually observed. Risk groups of HPgV include injection drug users, HIV-positive individuals, multi-transfused patients, hemodialysis patients, hemophiliacs, chronic liver disease patients and organ transplant recipients. The clinical significance of HPgV is not yet established and warrants further studies. Research on HPgV in the Philippines is scarce and has not been updated for over 10 years. There is no published data on HPgV prevalence in Filipino pediatric population specifically among risk groups like multi-transfused children with decompensated liver disease secondary to biliary cirrhosis and liver transplant pediatric patients. The lack of local data warrants conduct of this study.
\end{abstract}

Objective. To determine the presence of HPgV RNA, HPgV E2 antibody (anti-E2) and HBsAg among Filipino children with decompensated liver disease secondary to biliary cirrhosis (DBC) and liver transplant pediatric patients (LTP).

Methods. Included were 15 children with DBC and 15 LTP recruited from the Section of Pediatric Gastroenterology, Hepatology and Nutrition of the UP PGH. All patients' sera were tested for HPgV RNA by Real Time RT-PCR, $\mathrm{HPgV}$ anti-E2 by Enzyme-linked Immunosorbent Assay (ELISA) and hepatitis B surface antigen (HBsAg) by immunochromatographic test. Twenty age and sex matched children with no history of liver disease and blood transfusion served as controls.

Results. All patient and control samples were negative for HPgV RNA. HPgV anti-E2 was detected in 6 of 15 LTP, 5 of $15 \mathrm{DBC}$ and 1 of 20 controls. HBsAg was detected in 2 of 15 LTP, 5 of 15 DBC and 0 of 20 controls. Four patients (two LTP, two DBC) were positive for both HPgV anti-E2 and HBsAg.

Conclusion. This study showed that a proportion of liver transplant patients and those with decompensated biliary cirrhosis are positive for HPgV anti-E2, which indicates that these individuals previously had HPgV infection

Paper presented at the $21^{\text {st }}$ Biological Sciences Graduate Congress, December 15-16, 2016, Kuala Lumpur, Malaysia; at the 25th FAOBMB International Conference and 43 rd PSBMB Annual Convention, December 5-7, 2016, Manila, Philippines; and at the MEDEUREKA: Trends and Breakthroughs in Molecular Medicine, February 10, 2018, Manila, Philippines.

Corresponding author: Inah Marie C. Aquino

College of Medicine

University of the Philippines Manila

Ermita, Manila 1000, Philippines

Email: inahaquino18@gmail.com but is now resolved. Possible source of infection is infected blood from the blood transfusions, infected transplant organ or infected mother. Since routine HPgV screening is not yet recommended for the general population, blood donors and organ donors, the confirmation of exact source of infection may be difficult. Co-infection with $\mathrm{HBsAg}$ was also observed in both risk groups which suggests that at some point in time, these children were infected by both HPgV and $\mathrm{HBV}$ and also the possibility of simultaneous infection by the two viruses. This study provides preliminary 
data on the proportion of $\mathrm{HPgV}$ infection in Filipino children belonging to two of the HPgV risk groups. Studies with a larger and more significant sample size to determine $\mathrm{HPgV}$ prevalence as well as studies regarding the pathogenicity of $\mathrm{HPgV}$ are warranted. As this may provide basis for routine HPgV screening among risk groups and blood donations in the future.

Key Words: GBV-C, hepatitis G virus, HPgV, Filipino, pediatric, multi-transfused, transplant patients

\section{INTRODUCTION}

Human pegivirus ( $\mathrm{HPgV})$, formerly called Hepatitis G virus $(\mathrm{HGV})$ or $\mathrm{GB}$ virus $\mathrm{C}$, is an enveloped, spherical virus of about $50 \mathrm{~nm}$ in diameter belonging to the Flaviviridae family. ${ }^{1}$ It has a single stranded, positive sense RNA genome of $9.4 \mathrm{~kb}$ in length which contains a single long open reading frame (ORF) flanked by 5' and 3' untranslated regions. ${ }^{2}$ This open reading frame encodes for two envelope proteins $\left(\mathrm{E}_{1}\right.$ and $\left.\mathrm{E}_{2}\right), 3,4$ Hepatitis $\mathrm{C}$ virus $(\mathrm{HCV})$ is the closest relative of $\mathrm{HPgV}$ known to infect humans, they have the same genomic organization yet share only $25 \%$ sequence similarity. ${ }^{5} \mathrm{HPgV}$ predominantly replicates in peripheral blood mononuclear cells, mainly in B and $\mathrm{T}$ lymphocytes and bone marrow, and poorly in hepatocytes. ${ }^{6,7}$

$\mathrm{HPgV}$ is transmitted sexually, vertically (mother to infant) and parenterally or through exposure to infected blood. ${ }^{8}$ It shares common routes of transmission to other viruses such as Hepatitis $B$ virus (HBV), Hepatitis C virus $(\mathrm{HCV})$ and Human Immunodeficiency virus (HIV), thus co-infection is usually observed. ${ }^{8,9,10}$ Risk groups for $\mathrm{HPgV}$ include parenteral drug users, people living with HIV, men who have sex with men, multi-transfused patients, hemodialysis patients and haemophiliacs. ${ }^{11,12}$

The pathogenic mechanism of $\mathrm{HPgV}$ remains unclear. $\mathrm{HPgV}$ infection may last for months to years and can be detected by the presence of viral RNA in the blood. ${ }^{13}$ Clearance of $\mathrm{HPgV}$ is associated by the presence of specific antibodies against $\mathrm{HPgV}$ envelope protein $\mathrm{E} 2$ $(\mathrm{HPgV}$ anti-E2), moreover this is used as a marker for resolved infection. ${ }^{1,13}$

Multiple studies have explored the possible association of $\mathrm{HPgV}$ with known clinical diseases. At the early years of the discovery of $\mathrm{HPgV}$, it has been associated to nonA-E fulminant hepatitis but numerous studies designed to confirm this found no epidemiological associations. ${ }^{6,14,15}$ As mentioned, $\mathrm{HPgV}$ replicates in both $\mathrm{T}$ and $\mathrm{B}$ lymphocytes, in relation to this, several studies have reported association between $\mathrm{HPgV}$ viremia with an increased risk of nonHodgkin's lymphoma. ${ }^{13,16,17,18}$ Beneficial effects of $\mathrm{HPgV}$ viremia on HIV disease progression was explored by several groups and found higher CD4 cell counts, lower HIV viral loads and longer AIDs-free survival times for individuals with $\mathrm{HPgV}$ infection. ${ }^{19-22}$ However, other studies have contradicted these findings and observed no beneficial effects of $\mathrm{HPgV}$ infection in individuals living with HIV. ${ }^{23,24,25}$

Tucker et al. reported that $\mathrm{HPgV}$ has worldwide distribution and is found in the general population. ${ }^{26}$ Prevalence of $\mathrm{HPgV}$ viremia in healthy blood donors from developing countries ranges from $5 \%$ to $18.9 \%$ while in developed countries the prevalence of $\mathrm{HPgV}$ ranges from $0.5 \%$ to $5 \% .{ }^{1,27}$ Among the populations at high risk for infection, $\mathrm{HPgV}$ prevalence approaches $50 \% .{ }^{6,28,29}$ There are multiple variabilities in the evaluation of the prevalence of $\mathrm{HPgV}$ which includes different age groups, geographical regions, co-morbidities and sample size to name a few. The real burden of $\mathrm{HPgV}$ infection is not yet established and up to this day, the clinical significance of $\mathrm{HPgV}$ is not yet clear. Currently there is no recommendation regarding the risk for $\mathrm{HPgV}$ infection, no country has implemented routine $\mathrm{HPgV}$ screening in blood donors. ${ }^{1}$

In the Philippines, there is limited data on the prevalence of $\mathrm{HPgV}$ infection. In 2002, Dalmacio et al. published a prevalence data on $\mathrm{HPgV}$ infection in various risk groups and found $\mathrm{HPgV}$ prevalence in $1.2 \%$ volunteer blood donors, $8.0 \%$ on chronic liver patients, $3.4 \%$ hemodialysis patients and $6.2 \%$ multi-transfused patients. ${ }^{30}$ Since then, there has been no locally published literature on $\mathrm{HPgV}$. Moreover, there is no published data on $\mathrm{HPgV}$ prevalence in Filipino pediatric population specifically among risk groups like multi-transfused children with decompensated liver disease secondary to biliary cirrhosis and liver transplant pediatric patients. The lack of local data warrants conduct of this study.

This is a descriptive study on the presence of $\mathrm{HPgV} \mathrm{RNA}$, $\mathrm{HPgV}$ E2 antibodies (anti-E2) and HBV surface antigen (HBsAg) among Filipino children with decompensated liver disease due to biliary cirrhosis and liver transplant patients.

\section{METHODS}

\section{Study population and Ethics Clearance}

In a span of 1 year, Filipino patients aged 0-12 were recruited from the Section of Pediatric Gastroenterology, Hepatology and Nutrition of the Philippine General Hospital (PGH). Controls were recruited from the PGH Laboratory. Study groups included multi-transfused children with decompensated liver disease secondary to biliary cirrhosis, who had at least 2 previous blood transfusions ${ }^{31}$ and liver transplant patients, who was at least 6 months post operation. Controls were age and sex-matched children with no history of liver disease and blood transfusion.

Ethics approval was obtained from the University of the Philippines Manila - Research Implementation Development Office (RIDO) and Research Ethics Board (REB). After obtaining a written informed consent/assent from their parents/guardians, they were enrolled in the study. Only the patient, parent/guardian and patient's physician were informed of the results of this study. It was explained to the parent/guardian that due to the lack of established 
clinical significance of $\mathrm{HPgV}$ infection, there is currently no treatment for it. Appropriate medical care were provided by the physician in charge. The participant or the participant's parent or guardian can withdraw permission from using the blood samples of the subjects anytime.

\section{Blood Extraction and Serum Preparation}

Five $\mathrm{mL}$ of blood sample was taken from each patient during their routine blood extraction at PGH. Samples for the control group were from residual serum samples from patients with no history of liver disease and blood transfusion obtained from the PGH Laboratory. No additional blood extraction was done to patients in this group. Serum was separated from the whole blood within 6 hours of extraction, by centrifugation and was kept at $-80{ }^{\circ} \mathrm{C}$ until analysis.

\section{RNA Extraction for Real Time Reverse Transcription PCR}

Viral RNA was extracted from the serum samples using High Pure Viral Nucleic Acid Kit (Roche) following the manufacturer's instructions. ${ }^{32} 200 \mu \mathrm{l}$ serum was mixed with $200 \mu \mathrm{l}$ binding buffer and $50 \mu \mathrm{l}$ of proteinase $\mathrm{K}$ solution and was incubated for 10 minutes at $72^{\circ} \mathrm{C}$. After the addition of $100 \mu \mathrm{l}$ of binding buffer, the sample was transferred to a high filter tube and centrifuged for $1 \mathrm{~min}$ at $8000 \mathrm{x}$ g. After centrifugation, the filter tube was removed and transferred to a new collection tube, then $500 \mu \mathrm{l}$ inhibitor removal buffer was added to the upper reservoir of the filter tube and was centrifuged for $1 \mathrm{~min}$ at $8000 \mathrm{x}$ g. The same steps were repeated twice using $450 \mu \mathrm{l}$ wash buffer instead of inhibitor removal buffer. After centrifugation, the assembly was centrifuged for another 10 secs at maximum speed of $13,000 \times \mathrm{g}$ to remove any residual wash buffer. The filter tube was then transferred to a nuclease-free, sterile $1.5 \mathrm{ml}$ microcentrifuge tube. Finally, the $50 \mu \mathrm{l}$ of elution buffer was added to the upper reservoir of the filter tube and then centrifuged for $1 \mathrm{~min}$ at $8000 \mathrm{x}$ g to elute the viral nucleic acids. The extracted nucleic acid was stored in $-80^{\circ} \mathrm{C}$ freezer.

\section{Detection of HPgV RNA virus using HGV Real Time Reverse Transcription PCR kit}

$\mathrm{HPgV}$ RNA in serum was detected using HGV Real Time Reverse Transcription PCR Kit (Liferiver)(note HPgV was previously called HGV). ${ }^{33}$ The Real Time PCR system was Applied Biosystems 7500 Fast Real Time PCR system. The master reaction mix contains Super Mix for the specific amplification of $\mathrm{HPgV}$ RNA. The reaction was done in one step real time RT-PCR. HPgV RNA was transcribed into cDNA then a thermostable DNA polymerase was used to amplify the specific gene fragments by polymerase chain reaction. Fluorescence was emitted and measured by the real time system's optical unit. Detection of amplified $\mathrm{HPgV}$ DNA fragment was performed in fluorimeter channel FAM with the fluorescent quencher NFQ-MGB. This kit also includes internal control for identification of possible PCR inhibition and an external positive control defined as $1 \times 10^{7}$ copies $/ \mathrm{ml}$.

\section{Detection of HPgV RNA was performed following the manufacturer's protocol}

$20 \mu \mathrm{l}$ master mix containing $18 \mu \mathrm{l}$ super mix, $1 \mu \mathrm{l}$ enzyme mix and $1 \mu \mathrm{l}$ internal control was placed to each Real Time PCR reaction plate well. Separately $5 \mu$ each of RNA sample, positive and negative controls were added to different tubes. The running conditions were 1 cycle at $45^{\circ} \mathrm{C}$ for 10 minutes, 1 cycle at $95{ }^{\circ} \mathrm{C}$ for 15 minutes and 40 cycles at $95^{\circ} \mathrm{C}$ for 15 seconds, $60{ }^{\circ} \mathrm{C}$ for 1 minute with fluorescence measured at $60{ }^{\circ} \mathrm{C} .7500$ Software v 2.0.6 was used to analyze the obtained Real Time PCR data to determine absence or presence of $\mathrm{HPgV} \mathrm{RNA}$.

\section{Detection of HPgV E2 antibody}

$\mathrm{HPgV}$ E2 antibodies was detected using Human hepatitis $G$ virus antibody (IgG) ELISA kit (BioSource, San Diego CA) following manufacturer's instructions. ${ }^{34}$ In this kit, ELISA plate pre-coated with human $\mathrm{HPgV}$ E2 antigen, negative control and positive control for $\mathrm{HPgV} \mathrm{E} 2$ antigen were provided.

$10 \mu l$ each of the negative control, positive control and serum sample were added to different wells in the ELISA plates, then $100 \mu \mathrm{l}$ sample diluent was added to each well. A well for the blank, solely sample diluent, was also provided. Plate was covered with adhesive strip and was incubated for 45 minutes at $37{ }^{\circ} \mathrm{C}$. Then each well was aspirated and washed with $200 \mu \mathrm{l}$ wash buffer four times. The plate was inverted and blotted against clean paper towels. $100 \mu \mathrm{l}$ of Horseradish peroxidase (HRP)-conjugate was added to each well except to the blank well and was incubated for 30 minutes at $37^{\circ} \mathrm{C}$. The plate was then washed with wash buffer then inverted and blotted against clean paper towels. After that, $50 \mu 1$ of Substrate A and $50 \mu 1$ of Substrate B was added to each well and was incubated for 10 minutes at $37^{\circ} \mathrm{C}$. After incubation, $50 \mu \mathrm{l}$ of stop solution was added to each well. The optical density of each well within 10 minutes at $450 \mathrm{~nm}$ was determined.

\section{Interpretation of Result}

The calculation of the valence of human $\mathrm{HPgV}$ antibody (IgG) was done by comparing the sample well with the control. A cut-off value was defined as the average negative control value plus 0.2 . If ODsample < cut-off value then sample is negative for the antibody while if ODsample $\geq$ cut-off value then sample is positive for the antibody.

\section{Detection of HBV infection}

The serum sampleswere tested for the presence of Hepatitis $\mathrm{B}$ antigens using an $\mathrm{HBsAg}$ immunochromatographic test. This test utilizes the principle of immunochromatography. Test strips coated with colored polyclonal anti-HBsAgcolloidal gold conjugate complexes was dipped in serum 
sample until $1 \mathrm{~cm}$ high. Two visible lines in the test strip indicates presence of $\mathrm{HBsAg}$.

\section{RESULTS}

\section{Patients and control group}

In the span of 1 year, there were a total of 50 patients recruited in this study, 30 patients with liver disease, 15 patients with decompensated liver disease secondary to biliary cirrhosis and 15 post-liver transplant patients, and 20 controls (Table 1). Broken down into the two groups, those with decompensated liver disease, with 6 males and 9 females, were younger (median age: 1 year) compared to liver transplant patients, with 8 males and 7 females, (median age: 3 years). The control group consisted of 10 males and 10 females (median: 2 years).

Table 1. Summary of Patient and Control Group Data

\begin{tabular}{ccc} 
Group & No. of subjects & Age, median, years \\
DBC & 15 (6 males $/ 9$ females $)$ & 1 \\
LTP & 15 (8 males $/ 7$ females $)$ & 3 \\
Controls & $20(10$ males $/ 10$ females $)$ & 2 \\
\hline Total & 50 & -
\end{tabular}

LTP: liver transplant patients

DBC: decompensated liver disease secondary to biliary cirrhosis

\section{Detection of Human Pegivirus (HPgV) RNA}

Detection of HPgV RNA in the sera was done to determine active infection of $\mathrm{HPgV}$. Patient and control samples were negative for $\mathrm{HPgV}$ RNA (Table 2).

Table 2. Proportion of HPgV RNA, HPgV ANTI-E2 and HBsAG Positive Patients and Controls

\begin{tabular}{cccccc} 
Group & $\begin{array}{c}\text { No. of } \\
\text { Subjects }\end{array}$ & $\begin{array}{c}\text { No. of } \\
\text { HPgV } \\
\text { RNA(+) }\end{array}$ & $\begin{array}{c}\text { No. of } \\
\text { HPgV } \\
\text { anti-E2 (+) }\end{array}$ & $\begin{array}{c}\text { No. of } \\
\text { HBsAg } \\
(+)\end{array}$ & $\begin{array}{c}\text { HPgV anti-E2 } \\
\text { and HBsAg } \\
(+)\end{array}$ \\
\hline LTP & 15 & 0 & 6 & 2 & 2 \\
DBC & 15 & 0 & 5 & 5 & 2 \\
Controls & 20 & 0 & 1 & 0 & 0 \\
\hline Total & 50 & 0 & 12 & 7 & 4 \\
\hline
\end{tabular}

\section{Detection of Human Pegivirus E2 Antibody (HPgV anti-E2)}

Detection of the presence of the $\mathrm{HPgV}$ anti-E2 in the sera was done to determine resolved $\mathrm{HPgV}$ infection among the risk groups. $\mathrm{HPgV}$ anti-E2 was detected in 6 of 15 liver transplant patients, 5 of 15 decompensated liver disease secondary to biliary cirrhosis and 1 of 20 controls (Table 2).

\section{Detection of Hepatitis B Surface Antigen (HBsAg)}

Detection of HBsAg using immunochromatographic test was done to determine infection with hepatitis $B$ virus. HBsAg was detected in 2 of 15 liver transplant patients, 5 of 15 children with decompensated liver disease secondary to biliary cirrhosis and none from the controls (Table 2).

\section{Co-infection of HPgV and HBV}

$\mathrm{HPgV}$ share common routes of transmission to other viruses such as HBV. For the two risk groups, 2 from each tested positive for both $\mathrm{HPgV}$ anti-E2 and $\mathrm{HBsAg}$ (Table 2).

\section{DISCUSSION}

For more than a decade now, there have been no updates on the prevalence of $\mathrm{HPgV}$ infection in the Philippines. There is also no published data on $\mathrm{HPgV}$ infection among Filipino children specifically in multi-transfused children with decompensated liver disease secondary to biliary cirrhosis and post-liver transplant patients. In this study, 15 pediatric patients with decompensated liver disease secondary to biliary cirrhosis, 15 post-liver transplant pediatric patients and 20 controls were recruited to determine presence of $\mathrm{HPgV}$ infection.

To determine active $\mathrm{HPgV}$ infection, the presence of $\mathrm{HPgV}$ RNA in the blood was detected by reverse transcriptase PCR (RT-PCR). ${ }^{13}$ In this study, all patient and control samples were negative for $\mathrm{HPgV}$ RNA which indicates that none were infected with the virus at the time of blood extraction. To determine if these children had previous $\mathrm{HPgV}$ infection that resolved, ELISA was done to detect $\mathrm{HPgV}$ anti-E2. Several studies have shown that serum samples that are positive for the antibodies are negative for viral RNA and vice versa which makes $\mathrm{HPgV}$ anti-E2 a marker for resolved infection. ${ }^{35} \mathrm{HPgV}$ anti-E2 was detected in 6 of 15 liver transplant patients, 5 of 15 children with decompensated liver disease and 1 of 20 controls (Table 2). This indicates that there was previous $\mathrm{HPgV}$ infection in the $\mathrm{HPgV}$ anti-E2 positive children. Mechanism of viral clearance is still unclear at the moment and calls for further studies. Children from the two risk groups have had previous blood transfusions. They are at risk for $\mathrm{HPgV}$ infection since $\mathrm{HPgV}$ can be transmitted parenterally. $\mathrm{HPgV}$ is not routinely screened in blood donations and in organ donations thus infection from the transfusions and organ transplant is possible but is not documented. It is also possible that $\mathrm{HPgV}$ was transmitted vertically, through transmission of the virus from infected mothers. Serum from the mothers of the $\mathrm{HPgV}$ anti-E2 positive children can be tested for $\mathrm{HPgV}$ RNA and $\mathrm{HPgV}$ anti-E2 to help elucidate possible source of infection. However since collection of samples from the mothers is not within the scope of this study, this can be a recommendation for future studies. The $\mathrm{HPgV}$ anti-E2 positive control may have likely got the infection from infected mothers since there is no history of blood transfusion or liver disease.

In this study, HBV infection was determined by $\mathrm{HBsAg}$ using immunochromatographic test. HBV infection remains a major public health problem in the Philippines despite vaccination efforts and routine screening in blood donations. An estimated 7.3 million adult Filipinos (16.7\%) are infected which makes the country hyperendemic for hepatitis $\mathrm{B} .{ }^{36}$ Children are also at risk for $\mathrm{HBV}$ infection, 
most individuals that develop chronic hepatitis B acquire the infection at birth or during early childhood. ${ }^{36}$ The risk groups in this study are at risk for HBV infection as well from the multiple blood transfusions and other parenteral routes, infected needles, catheters, exposure to infected fluids and infected organ. $\mathrm{HBsAg}$ was detected in 2 of 15 liver transplant patients, 5 of 15 children with decompensated liver disease secondary to biliary cirrhosis and none from the controls (Table 2). For the two risk groups, 2 from each tested positive for both $\mathrm{HPgV}$ anti-E2 and $\mathrm{HBsAg}$ (Table 2). This suggests that at some point in time, these children were infected by both $\mathrm{HPgV}$ and $\mathrm{HBV}$. This also suggests the possibility of simultaneous infection by the two viruses. ${ }^{30}$ Possible source of HBV infection of these children is from an infected mother.

Results of this study were relayed to the parent/ guardian of the child and to the patient's physician in charge. There is currently no recommended treatment for $\mathrm{HPgV}$ infection. There is still no clear evidence that $\mathrm{HPgV}$ can cause human disease and there is also no established association with known diseases. ${ }^{1}$ Appropriate medical care specifically for those who tested positive for HBsAg was provided by their respective physicians.

This is the first study to report data on the presence of $\mathrm{HPgV}$ infection among Filipino children belonging to two of the $\mathrm{HPgV}$ risk groups: decompensated liver disease secondary to biliary cirrhosis and liver transplant pediatric patients. However, due to the small sample size, the results is not a good representation of the prevalence rate of $\mathrm{HPgV}$ infection among children belonging to the risk groups. Further studies with a larger and more significant sample size are recommended to be able to determine prevalence of $\mathrm{HPgV}$ infection among these groups and eventually in other pediatric patients under the other risk groups. In addition, the mothers of the children may also be included in the study since they are possible sources of infection. Studies regarding the pathogenicity of $\mathrm{HPgV}$ in Filipino children belonging to the risks groups are also recommended.

\section{CONCLUSIONS}

This study showed that a proportion of liver transplant patients and those with decompensated biliary cirrhosis are positive for $\mathrm{HPgV}$ anti-E2, which indicates that these individuals previously had $\mathrm{HPgV}$ infection but is now resolved. Possible source of infection is infected blood from the blood transfusions, infected organ or infected mother. Since routine $\mathrm{HPgV}$ screening is not yet recommended for the general population, blood donors and organ donors, the confirmation of exact source of infection may be difficult. Co-infection with $\mathrm{HBsAg}$ was also observed in both risk groups which suggests that at some point in time, these children were infected by both $\mathrm{HPgV}$ and $\mathrm{HBV}$ and also the possibility of simultaneous infection by the two viruses. This study provides preliminary data on the proportion of $\mathrm{HPgV}$ infection in Filipino children belonging to two of the $\mathrm{HPgV}$ risk groups. Future studies with a larger and more significant sample size to determine $\mathrm{HPgV}$ prevalence as well as studies regarding the pathogenicity of $\mathrm{HPgV}$ are warranted. Such studies may provide basis for routine $\mathrm{HPgV}$ screening among risk groups and blood donations in the future.

\section{Acknowledgments}

The authors would like to thank the study participants and the Section of Pediatric Gastroenterology, Hepatology and Nutrition of the UP PGH and the PGH laboratory for their assistance in collection of patient and control samples. The authors would also like to thank the Department of Biochemistry and Molecular Biology, College of Medicine, University of the Philippines Manila for lending their laboratory and resources for the experiments. The authors would also like to express their gratitude to the Philippine Council for Health Research and Development-DOST for the support.

\section{Statement of Authorship}

All authors have approved the final version submitted.

\section{Author Disclosure}

All authors declared no conflicts of interest.

\section{Funding Source}

This study is funded by the Philippine Council for Health Research and Development-DOST.

\section{REFERENCES}

1. Marano G, Franchini M, Farina B, Piccinini V, Pupella S, Vaglio $\mathrm{S}$, et al. The human pegivirus: A new name for an "ancient" virus. Can transfusion medicine come up with something new? Acta Virol. 2017 Jan; 61(4):401-12.

2. Ghai RR, Sibley SD, Lauck M, Dinis JM, Bailey AL, Chapman CA, et al. Deep sequencing identifies two genotypes and high viral genetic diversity of human Pegivirus (GB Virus C) in rural Ugandan patients. J Gen Virol. 2013 Dec; 94(Pt 12):2670-8.

3. Katayama K, Kageyama T, Fukushi S, Hoshino FB, Kurihara C, Ishiyama N, et al. Full-length GBV-C/HGV genomes from nine Japanese isolate: characterization by comparative analyses. Arch Virol. 1998; 143(6):1063-75.

4. Hammad AM, Zaghloul MH. Hepatitis G virus infection in Egyptian children with chronic renal failure (single centre study). Ann Clin Microbiol Antimicrob. 2009 Dec; 8:36.

5. Di Bisceglie AM. Hepatitis G virus infection: a work in progress. Ann Intern Med.1996 Nov; 125(9):772-3.

6. Reshetnyak VI, Karlovich TI, Ilchenko LU. Hepatitis G virus. World J Gastroenterol. 2008 Aug; 14(30):4725-34.

7. George SL, Varmaz D, Stapleton JT. GB virus $\mathrm{C}$ replicates in primary T and B lymphocytes. J Infect Dis. 2006 Feb; 193(3):451-4.

8. Bhattarai N, Stapleton JT. GB virus C: the good boy virus? Trends Microbiol. 2012 Mar; 20(3):124-30.

9. Mphahlele MJ, Lau GK, Carman WF. HGV: the identification, biology and prevalence of an orphan virus. Liver. 1998 Jun;18(3): 143-55.

10. Barusruk S, Urwijitaroon Y. High prevalence of HGV coinfection with HBV or HCV among northeastern Thai blood donors. Southern Asian J Trop Med Public Health. 2006 Mar; 37(2):289-93. 
11. Lefrere JJ, Roudot-Thoraval F, Morand-Joubert L, Brossard Y, Parnet-Mathieu F, Mariotti M, et al. Prevalence of GB virus type $\mathrm{C} /$ hepatitis $\mathrm{G}$ virus RNA and of anti-E2 in individuals at high or low risk for blood-borne or sexually transmitted viruses: evidence of sexual and parenteral transmission. Transfusion. 1999 Jan; 39(1): 83-94.

12. Vahidnia F, Petersen M, Rutherford G, Busch M, Assmann S, Stapleton JT, et al. Transmission of GB Virus Type C via Transfusion in a Cohort of HIV-Infected Patients. J Infect Dis. 2012 May; 205(9):1436-42.

13. Krajden M, Yu A, Braybrook H, Lai AS, Mak A, Chow R, et al. $\mathrm{GBV}-\mathrm{C} /$ hepatitis $\mathrm{G}$ virus infection and non-Hodgkin lymphoma: A case control study. Int J Cancer. 2010 Jun; 126(12):2885-92.

14. Sheng L, Soumillion A, Beckers N, Wu CG, Verslype C, Nevens F, et al. Hepatitis $\mathrm{G}$ virus infection in acute fulminant hepatitis: prevalence of HGV infection and sequence analysis of a specific viral strain. J Viral Hepat. 1998 Sep; 5(5):301-6.

15. Anastassopoulou CG, Delladetsima JK, Anagnostopoulos G, Katsoulidou A, Papachristopoulos A, Tassopoulos NC, et al. Fulminant hepatic failure in a pediatric patient with active GB virus C (GBV-C)/hepatitis G virus (HGV) infection. Hepatol Res. 2002 Jun; 23(2):85-9.

16. Chivero ET, Stapleton JT. Tropism of human pegivirus (formerly known as $\mathrm{GB}$ virus $\mathrm{C} /$ hepatitis $\mathrm{G}$ virus) and host immunomodulation: insights into a highly successful viral infection. J Gen Virol. 2015 Jul; 96(Pt 7):1521-32.

17. Chang CM, Stapleton JT, Klinzman D, McLinden JH, Purdue MP, Katki HA, et al. GBV-C infection and risk of NHL among U.S. adults. Cancer Res. 2014 Oct; 74(19):5553-60.

18. Fama A, Xiang J, Link BK, Allmer C, Klinzman D, Feldman AL, et al. Human Pegivirus infection and lymphoma risk and prognosis: a North American study. Br J Haematol. 2018 Sep; 182(5):644-53.

19. Xiang J, Wunschmann S, Diekema DJ, Klinzman D, Patrick $\mathrm{KD}$, George SL, et al. Effect of coinfection with $\mathrm{GB}$ virus $\mathrm{C}$ on survival among patients with HIV infection. N Engl J Med. 2001 Sep; 345(10):707-14.

20. Tillmann HL, Heiken H, Knapik-Botor A, Heringlake S, Ockenga J, Wilber JC, et al. Infection with GB virus $\mathrm{C}$ and reduced mortality among HIV-infected patients. N Engl J Med. 2001 Sep; 345(10): $715-24$.

21. 21. Williams CF, Klinzman D, Yamashita TE, Xiang J, Polgreen PM, Rinaldo C, et al. Persistent GB virus $\mathrm{C}$ infection and survival in HIVinfected men. N Engl J Med. 2004 Mar; 350(10):981-90.

22. N'Guessan KF, Anderson M, Phinius B, Moyo S, Malick A, Mbangiwa T, et al. The impact of Human Pegivirus on CD4 cell count in HIVpositive persons in Botswana. Open Forum Infect Dis. 2017 Oct; 4(4):ofx222.
23. Björkman P, Flamholc L, Nauclér A, Molnegren V, Wallmark $\mathrm{E}$, Widell A, et al. GB virus $\mathrm{C}$ during the natural course of HIV-1 infection: viremia at diagnosis does not predict mortality. AIDS. 2004 Apr; 18(6):877-86.

24. Quiros-Roldan E, Maroto MC, Torti C, Moretti F, Casari S, Pan A, et al. No evidence of beneficial effect of GB virus type $\mathrm{C}$ infection on the course of HIV infection. AIDS. 2002 Aug; 16(10):1430-1.

25. Birk $M$, Lindbäck $S, L i d m a n C$. No influence of $G B$ virus $C$ replication on the prognosis in a cohort of HIV-1-infected patients. AIDS. 2002 Dec; 16(18):2482-5.

26. Tucker TJ, Smuts HE. Review of the epidemiology, molecular characterization and tropism of the hepatitis $\mathrm{G}$ virus/ GBV-C. Clin Lab. 2001; 47(5-6):239-48.

27. Mohr EL, Stapleton JT. GB virus type C interactions with HIV: the role of envelope glycoproteins. J Viral Hepat. 2009 Nov; 16(11): 757-68.

28. Schwarze-Zander C, Blackard JT, Rockstroh JK. Role of GB virus C in modulating HIV disease. Expert Rev Anti Infect Ther. 2012 May; 10(5):563-72.

29. Dawson GJ, Schlauder GG, Pilot-Matias TJ, Thiele D, Leary TP, Murphy P, et al. Prevalence studies of GB virus-C infection using reverse transcriptase-polymerase chain reaction. J Med Virol. 1996 Sep; 50(1):97-103.

30. Dalmacio LM, Domingo EO, Libranda-Ramirez BD, RicaforteCampos JD. Prevalence of hepatitis G virus (HGV) infection among risk groups in the Philippines. Acta Med Philipp. 2002; 38(2):24-36.

31. Thakral B, Saluja K, Sharma RR, Marwaha N. Red cell alloimmunization in a transfused patient population: a study from a tertiary care hospital in north India. Hematology. 2008 Oct;13(5):313-8.

32. High Pure Viral Nucleic Acit kit: Procedure [Internet]. 2008 [cited 2015 Apr 15]. Available from: https://lifescience.roche.com/ documents/High-Pure-Viral-Nucleic-Acid-Kit.pdf.

33. HGV Real Time RT-PCR Kit User Manual [Internet]. 2012 [cited 2015 Apr 16]. Available from: https://www.biosb.com/wp-content/ uploads/HR-0012-01-HGV-Real-Time-RT-PCR-Kit.pdf.

34. Human hepatitis $\mathrm{G}$ virus (HGV) antibody ( $\mathrm{IgG})$ ELISA kit manual. BioSource, San Diego CA.

35. Sathar M, Soni P, York D. GB virus C/hepatitis G virus (GBV-C/ HGV): still looking for a disease. Int J Exp Pathol. 2000 Oct; 81(5):305-22.

36. Gish RG, Sollano JD Jr, Lapasaran A, Ong JP. Chronic hepatitis $\mathrm{B}$ virus in the Philippines. J Gastroenterol Hepatol.2016 May; 31(5):945-52. 